\title{
Modelo Preditivo para Cesariana com Uso de Fatores de Risco
}

\author{
Predictive Model using Risk Factors for Cesarean Section \\ Alfredo de Almeida Cunha, Margareth Crisóstomo Portela \\ Abés Mahomed Amed, Luiz Camano
}

\begin{abstract}
RESUMO
Objetivos: Identificar fatores anteparto relacionados à ocorrência de cesariana. Construir modelo preditivo de cesariana.

Pacientes e Métodos: Foram estudados todos os 843 partos assistidos em unidade obstétrica de nivel III, no periodo de junho de 1993 a novembro de 1994.

$O$ delineamento do estudo foi de coorte do tipo retrospectivo. O critério de inclusão foi de recém-nascido vivo pesando $1.000 \mathrm{~g}$ ou mais. A variável dependente foi cesariana, dicotomizada como presente ou ausente. As variáveis independentes foram os fatores anteparto relacionados à cesariana. Para a construção do modelo foi utilizada a regressão logística.

Resultados: O modelo multivariado mostrou risco de cesariana de acordo com as seguintes variáveis independentes: adolescência (idade inferior a 20 anos), odds ratio $(O R)=0,396$; idade materna igual ou superior a 28 anos, $O R=2,133$; antecedente de parto normal, $O R=$ 0,626 ; antecedente de cesariana $O R=4,576$; assistência pré-natal, $O R=2,346$; apresentação pélvica, $O R=4,174$; gemelaridade $O R=14,065$; hemorragia da segunda metade da prenhez, $O R=28,189$; pré-eclampsia leve, $O R=2,180$; pré-eclampsia grave, $O R=16,738$; hipertensão arterial crônica, $O R=4,927$, e outras intercorrências maternas, $O R=2,012$. O modelo matemático mostrou concordância entre a probabilidade prevista e a resposta observada em 82,3\%, o que indica sua eficiência.

Conclusões: Foram identificados 12 fatores anteparto relacionados à ocorrência de cesariana. Foi possivel construir modelo preditivo de cesariana utilizando os fatores de risco anteparto identificados no presente estudo.
\end{abstract}

PALAVRAS-CHAVE: Cesariana. Modelo preditivo. Complicações da gravidez. Resolução da gravidez.

\section{Introdução}

Parto cesáreo (ou cesariana) é definido como o nascimento de um feto através de duas incisões: na parede abdominal (laparotomia) e na parede uterina (histerotomia). Esta definição não inclui a retirada do feto da cavidade abdominal no caso de rotura uterina ou no caso de prenhez abdominal ${ }^{1}$.

Departamento de Obstetrícia da Escola Paulista de Medicina, Universidade Federal de São Paulo e Disciplina de Obstetrícia da Faculdade de Medicina, Universidade do Estado do Rio de Janeiro

Correspondência:

Alfredo de Almeida Cunha

Rua Carlos Vasconcelos, 76 - ap 1402 - Tijuca

20521-050 - Rio de Janeiro - RJ

Tel.: (21) 2204-0867
A incidência de cesariana vem crescendo ao longo do tempo desde o início do século, quando era de 0,6\% na Maternidade de Chicago em 1910, sofrendo um aumento de cinco vezes (3\%) em 1928. Em anos subseqüentes aumentou de forma dramática nos Estados Unidos da América, elevandose de 4,5\% em 1965 para 16,5\% em 1980 e atingindo o máximo de $24,7 \%$ em 1988 , o que não foi acompanhado por todos os países. Por exemplo, dados disponiveis de $1985^{2}$ permitem comparar Estados Unidos da América (22,7\%), Canadá (19\%), Itália $(15,8 \%)$, Dinamarca (13\%), Suécia (12\%), Noruega $(12,5 \%)$, Inglaterra (10\%), Nova Zelândia $(10 \%)$ e Japão (7\%).

A cesariana tem tido suas indicações ampliadas em todo o mundo na intenção de se obter melhores resultados maternos e perinatais, obje- 
tivo nem sempre conseguido, motivando preocupação para sua redução. Na realidade, a diminuição da mortalidade perinatal deve-se principalmente aos avanços tecnológicos da obstetrícia e da assistência neonatal ${ }^{3}$.

A proposta de que a alta incidência de cesariana justifica-se pela redução na morbidade e mortalidade materna e perinatal não está comprovada. Pelo contrário, um estudo recente de 12 países concluiu que uma grande proporção de intervenções foram desnecessárias ou proporcionaram benefício mínimo. O aumento da incidência de cesariana foi associado à elevação dos riscos devidos à anestesia e morbidade operatória para a mãe e o recém-nascido, acrescidos dos riscos em partos futuros ${ }^{4}$.

No Brasil, notou-se uma continuação do aumento da incidência de cesariana. Na Escola Paulista de Medicina, por exemplo, as taxas evoluíram de 25,5\% em 1975 para 37,9\% em 1981, justificável, no entanto, pelo atendimento de população de alto risco ${ }^{5}$. A situação vem merecendo a atenção dos especialistas e das autoridades sanitárias brasileiras, podendo-se citar sociedades médicas, como a Federação Brasileira das Sociedades de Ginecologia e Obstetrícia (FEBRASGO, 1994) ${ }^{6}$ que têm divulgado o incentivo ao parto normal como parte do programa "Maternidade Segura" da Organização Mundial de Saúde (adotado pelo Ministério da Saúde do Brasil), e o Conselho Federal de Medicina (1997), em publicação intitulada: "Cesariana: uma epidemia invisivel". Cunha et al. ${ }^{7}$ expressaram recentemente sua preocupação com a alta incidência de cesariana no Brasil (32\% no Sistema Único de Saúde), considerando um problema de saúde pública.

Visto, sucintamente, o problema da incidência de cesariana é relevante qualquer instrumento que se disponha a estudá-la, constituindo instrumento de avaliação da qualidade da assistência prestada e da utilização da informação obtida para o planejamento em saúde. Dentre estes, os modelos preditivos permitem identificar os fatores de risco associados à ocorrência da cesariana. Os modelos preditivos (matemáticos) podem seguir vários modelos biológicos, que dependem da população estudada (exemplo, gravidez de alto ou baixo risco) e das diferentes condutas e ambientes de assistência. Assim, podemos construir modelos preditivos baseados em fatores identificados antes do parto e outros utilizando aqueles que surgem durante o parto (modelos biológicos), conforme utilizado por Hin et al. ${ }^{8}$.

O presente estudo epidemiológico teve como objetivo identificar fatores de risco anteparto para ocorrência de operação cesariana e a construção de um modelo preditivo que foi o instrumento de avaliação da probabilidade de cesariana para cada paciente da população em estudo, a maioria de gravidez de alto risco, assistida em serviço universitário.

\section{Pacientes e Métodos}

A amostra constou da análise de todos os 843 partos cesáreos e vaginais com recém-nascidos vivos (8 partos gemelares), de 835 pacientes assistidas no período de junho de 1993 a novembro de 1994 (18 meses) na maternidade do Hospital Universitário Pedro Ernesto da Universidade do Estado do Rio de Janeiro (HUPE/UERJ) que atenderam ao critério de inclusão, que foi de concepto com peso mínimo de 1.000 gramas (constituindo a população alvo).

As pacientes internadas no referido periodo foram encaminhadas do ambulatório pré-natal do HUPE/UERJ e do restante da rede pública. O HUPE/ UERJ, devido ao limite de sua capacidade, tem como critério matricular pacientes de risco elevado, tais como adolescentes (idade até 19 anos), adultas portadoras de alguma intercorrência clínica ou obstétrica e as com idade igual ou superior a 35 anos. O hospital integra sistema de referência e contra-referência, encaminhando para os postos de saúde e maternidades de nivel secundário (baixo risco) as pacientes que não necessitem de atendimento terciário do hospital universitário e aceitando encaminhamento de pacientes de risco elevado.

Além disso, existe entrosamento profissional mediante participação de médicos e professores do hospital no atendimento em toda a rede e troca de informações, treinamento e experiência por meio de eventos científicos, garantindo, dessa forma, consenso entre os profissionais.

O delineamento do estudo foi de coorte, do tipo retrospectivo, em que a variável dependente foi cesariana e as variáveis independentes foram os fatores de risco observados no periodo anteparto.

A identificação de fatores de risco anteparto para cesariana implicou estudo observacional do tipo coorte. O estudo foi retrospectivo devido à utilização de base de dados gerada pela internação das pacientes anterior ao mesmo. Alguns fatores poderiam ser identificados na primeira consulta, tais como idade materna, antecedente de partos normais ou cesáreos.

Outros fatores, entretanto, seriam caracterizados ao longo da gestação, dentre eles idade gestacional, assistência pré-natal, apresentação, amniorrexe prematura, gemelaridade e hemorragia da segunda metade da prenhez. 
Finalmente, um terceiro grupo compreenderia as doenças clínicas associadas à gestação, como os estados hipertensivos sob todas as formas e outras intercorrências (aqui incluídas cardiopatia, nefropatia, colagenose, diabete, infecção urinária).

A coorte (população em estudo) foi do tipo dinâmico, sendo que a inclusão no estudo era feita no momento do parto, quando era concluída a observação pela caracterização da via do parto. Foram observados 843 partos de recém-nascidos vivos de 835 pacientes ( 8 partos gemelares), aqui incluídos todos os que atenderam ao critério de inclusão.

O critério de inclusão de pacientes no estudo foi o nascimento de concepto vivo com peso mínimo de $1.000 \mathrm{~g}$. A exclusão de feto morto deveu-se à possibilidade de conduta diferente, o que iria constituir vício de seleção. O peso de $1.000 \mathrm{~g}$ é marco internacional para comparação de resultados perinatais, e é o limite abaixo do qual a conduta obstétrica pode modificar-se pelas condições de assistência neonatal, por vezes com mau prognóstico. Todas as pacientes atendidas no período do estudo e que atendiam ao critério de inclusão foram incluídas. Entretanto, pelas limitações de ser um estudo retrospectivo, limitamo-nos às variáveis que nos interessaram diretamente para a construção do modelo. Reconhecemos que neste momento surge um vício ("bias") do estudo, o que vai limitar a eficiência do modelo preditivo. Certamente, ele não terá eficiência de $100 \%$ (concordância entre o predito e o observado), devido à falta de outras variáveis não consideradas.

A análise estatística foi desenvolvida em três etapas: univariada, bivariada e multivariada. A análise univariada consta da verificação da base de dados e da observação da distribuição das variáveis (estatística descritiva). A análise bivariada consta da observação da associação de cada uma das variáveis independentes, (fatores de risco) com a variável dependente (cesariana). As variáveis independentes, para serem analisadas como fatores de risco, deveriam obedecer ao modelo biológico.

Os testes estatísticos utilizados na análise bivariada incluíram o teste do $\chi^{2}$, para comparação de proporções, e teste $t$ de Student, para comparação de médias.

$\mathrm{Na}$ presença de fator de risco dicotômico (presente ou ausente) e de cesariana também dicotômica (presente ou ausente), foi avaliado o risco de ocorrência de cesariana sob forma de razão de chances (OR $=$ odds ratio). A seleção das variáveis para inclusão no modelo multivariado foi a plausibilidade biológica dos fatores de risco e a seleção estatística com margem de erro alfa de $5 \%$ (valor $\mathrm{p}<0,05$ ).

$\mathrm{Na}$ análise multivariada todas as variáveis foram analisadas simultaneamente. No caso presente, como a variável dependente é categórica e dicotômica, está indicada a técnica de regressão logística, que aceita como variáveis independentes aquelas de natureza categórica, bem como as numéricas. O resultado final da análise foi a construção de modelo preditivo que calcula a probabilidade de cesariana na dependência dos fatores de risco.

O presente estudo foi aprovado pela Comitê de Ética em Pesquisa do Hospital Universitário Pedro Ernesto da Universidade do Estado do Rio de Janeiro.

\section{Resultados}

A Tabela 1 mostra a distribuição das variáveis numéricas, notando-se 25 anos como média (e mediana) da idade materna, com variação de 12 a 47 anos, sendo a idade mais freqüente 17 anos. A idade gestacional teve média de 38 semanas, e a mais freqüente foi 40 semanas. Em relação aos partos anteriores, houve variação de 0 a 8 partos normais e de 0 a 3 cesarianas. Carateriza-se a população pela amplitude da idade materna, sendo, entretanto, mais freqüente a idade mais jovem. As gestações foram, na média, a termo (38 semanas). Não houve antecedentes de multiparidade extrema (acima de 10 partos). A população caracterizouse por apresentar antecedentes de cesariana.

Tabela 1 - Distribuição das variáveis numéricas $(n=843)$.

\begin{tabular}{lccc}
\hline Variável & Média & $\begin{array}{c}\text { Desvio } \\
\text { padrão }\end{array}$ & Amplitude \\
\hline Idade materna (anos) & 25,7 & 7,5 & $12-47$ \\
Idade gestacional (semanas) & 38,9 & 3,1 & $20-45$ \\
Antecedente de parto normal (n) & 1 & 1,5 & $0-8$ \\
Antecedente de parto cesáreo $(\mathrm{n})$ & 0,2 & 0,5 & $0-3$ \\
\hline
\end{tabular}

A Tabela 2 mostra a distribuição das variáveis categóricas, consideradas de forma dicotômica, sendo considerado fator de risco a presença da variável. Esta codificação se torna necessária para a futura análise multivariada. Para fins da modelagem matemática foram considerados diferentes pontos de corte da idade materna, aos 20, 28 e aos 35 anos. Já os demais fatores, pela própria natureza categórica, foram considerados apenas como presentes ou ausentes. Notamos na lista das variáveis independentes (= fatores de risco), aqui incluídas, além da idade da paciente, assistência pré-natal, apresentação pélvica, amniorrexe prematura, gemelaridade, 
hemorragia da $2^{\mathrm{a}}$ metade da gravidez, préeclampsia leve, pré-eclampsia grave, hipertensão crônica e outras intercorrências maternas (consideradas em grupo pela baixa incidência de cada uma e referindo-se a outras complicações não especificadas, a saber: diabete melito, cardiopatias, nefropatias, doenças do colágeno e infecção urinária).

A Tabela 3 mostra a associação das variáveis numéricas com o desfecho (cesariana), por comparação de médias. Começa aqui a análise bivariada (ou inferencial). Nota-se associação direta da idade materna e do antecedente de cesariana com o risco de cesariana. Já a idade gestacional e o antecedente de partos normais mostram associação inversa (valor de $t$ negativo). A idade gestacional apresentou valor p de 0,0712 , que não seria estatisticamente significativo. Entretanto, na construção do modelo multivariado, devem ser consideradas variáveis com valor $\mathrm{p}$ de até 0,25.
Tabela 2 - Distribuição das variáveis categóricas em porcentagens $(n=843)$ (categorias: presente/ausente).

\begin{tabular}{lc}
\hline Variável & $\%$ \\
\hline Cesariana & 29,3 \\
Idade menor que 20 anos & 26,3 \\
Idade > 35 anos & 17,0 \\
idade > 28 anos & 38,7 \\
Assistência pré-natal & 87,8 \\
Apresentação pélvica & 3,0 \\
Amniorrexe prematura & 22,6 \\
Gemelaridade & 1,0 \\
Hemorragia 2a metade & 1,5 \\
Pré-eclampsia leve & 6,5 \\
Pré-eclampsia grave & 6,0 \\
Hipertensão crônica & 7,5 \\
Intercorrência materna outra & 6,4 \\
\hline
\end{tabular}

Tabela 3 - Associação das variáveis numéricas com a cesariana. Comparação das médias das variáveis contínuas entre os grupos de estudo e controle.

\begin{tabular}{lrrrr}
\hline Variável & Cesariana & & \\
& Presente & Ausente & $\mathbf{t}$ & $\mathbf{p}$ \\
\hline Idade materna (anos) & $28,6 \pm 7,2$ & $24,6 \pm 7,3$ & 7,2 & $<0,0001$ \\
Idade gestacional (sem) & $37,9 \pm 3,2$ & $38,4 \pm 3,0$ & $-1,8$ & 0,0712 \\
Antecedente parto normal (n) & $0,6 \pm 1,2$ & $1,1 \pm 1,6$ & $-5,3$ & 0,0001 \\
Antecedente parto cesáreo $(\mathrm{n})$ & $0,4 \pm 0,7$ & $0,06 \pm 0,2$ & 8,3 & 0,0001 \\
\hline
\end{tabular}

A Tabela 4 mostra a associação das variáveis categóricas com o desfecho, mediante utilização da razão de chances (OR) como medida de risco, continuando a análise bivariada, agora com a utilização de medida de efeito do fator de risco sobre o desfecho. Observou-se associação da ida- de materna com a cesariana nos diferentes pontos de corte utilizados. Todas as demais variáveis apresentaram associação com a cesariana, sendo candidatas, portanto, a serem incluídas na análise pelo modelo multivariado.

Tabela 4 - Razão de chances das variáveis categóricas para cesariana. A categoria de referência é a ausência do fator.

\begin{tabular}{lcccc}
\hline Fator & OR & IC 95\% & $\boldsymbol{\chi}^{2}$ & $\mathbf{p}$ \\
\hline Idade menor que 20 anos & 0,3 & $0,2-0,5$ & 30,3 & $<0,001$ \\
Idade $>35$ anos & 2,2 & $1,4-3,2$ & 18,1 & $<0,001$ \\
Idade $>28$ anos & 2,4 & $1,7-3,3$ & 33,9 & $<0,001$ \\
Gemelaridade & 7,3 & $1,3-54,0$ & Fisher & 0,009 \\
Hipertensão crônica & 4,2 & $2,4-7,5$ & 33,4 & 0,001 \\
Apresentação pélvica & 3,2 & $1,3-7,7$ & 8,8 & 0,003 \\
Assistência pré-natal & 2,4 & $1,3-4,5$ & 10,7 & 0,001 \\
Pré-eclampsia leve & 2,1 & $1,1-3,8$ & 7,4 & 0,006 \\
Pré-eclampsia grave & 11,6 & $5,4-25,5$ & 68,4 & 0,001 \\
Amniorrexe prematura & 0,6 & $0,4-0,9$ & 6,1 & 0,013 \\
Hemorragia da segunda metade & 27,7 & $3,6-587,7$ & Fisher & $<0,001$ \\
Intercorrência materna outra & 2,3 & $1,3-4,3$ & 9,8 & 0,002 \\
\hline
\end{tabular}


A Tabela 5 mostra o modelo logístico final, obtido após processo durante o qual foram testados 7 modelos. Este modelo definiu as variáveis associadas com a cesariana depois de testados 6 outros modelos, dos quais este foi o mais significativo. Nele, a idade materna inferior a 20 anos mostrou-se como fator de proteção para a cesariana, bem como o antecedente de parto normal (parâmetro com sinal negativo). As demais variáveis foram de risco para cesariana, sendo o ponto de corte para a idade materna significativo aos 28 anos. A intercorrência materna resume grupo de complicações clínicas outras, aqui consideradas em grupo: cardiopatia, nefropatia, infecção urinária, colagenose e diabete. A análise seguiu o modelo de máxima verossimilhança, técnica estatística cujo resultado segue uma distribuição $\chi^{2}$, o que facilita sua interpretação em relação ao valor $\mathrm{p}$ (significa que o modelo foi altamente significativo do ponto de vista estatístico).

Tabela 5 - Modelo final. Estratificação de idade materna com maior poder de predição e eliminação das variáveis não significativas (idade gestacional e amniorrexe prematura).

\begin{tabular}{|c|c|c|c|c|c|c|}
\hline \multirow{2}{*}{$\frac{\text { Variável }}{\text { Intercepto }}$} & \multirow{2}{*}{$\begin{array}{c}\text { Estimativa do } \\
\text { parâmetro }\end{array}$} & \multirow{2}{*}{$\begin{array}{c}\text { Erro padrão } \\
0,3\end{array}$} & \multirow{2}{*}{$\begin{array}{c}\text { Valor } \mathbf{p} \\
<0,01\end{array}$} & \multirow[t]{2}{*}{ Razão de chances } & \multicolumn{2}{|c|}{$\begin{array}{l}\text { Intervalo de } \\
\text { confiança } 95 \%\end{array}$} \\
\hline & & & & & & \\
\hline Idade materna $<20$ & $-0,9$ & 0,2 & $<0,01$ & 0,3 & 0,2 & 0,6 \\
\hline Idade materna $>28$ & 0,7 & 0,2 & $<0,01$ & 2,1 & 1,3 & 3,2 \\
\hline Antecedente de parto normal & $-0,4$ & 0,08 & $<0,01$ & 0,6 & 0,5 & 0,7 \\
\hline Antecedente de cesariana & 1,5 & 0,2 & $<0,01$ & 4,5 & 3,0 & 6,9 \\
\hline Assistência pré-natal & 0,8 & 0,3 & 0,01 & 2,3 & 1,1 & 4,6 \\
\hline Apresentação pélvica & 1,4 & 0,5 & $<0,01$ & 4,1 & 1,5 & 11,5 \\
\hline Gemelaridade & 2,6 & 1,0 & $<0,01$ & 14,0 & 1,9 & 102,0 \\
\hline Hemorragia $2^{a}$ metade & 3,3 & 1,1 & $<0,01$ & 28,1 & 2,8 & 277,3 \\
\hline Pré-eclampsia leve & 0,7 & 0,3 & 0,01 & 2,1 & 1,1 & 4,1 \\
\hline Pré-eclampsia grave & 2,8 & 0,4 & $<0,01$ & 16,7 & 7,4 & 37,6 \\
\hline Hipertensão crônica & 1,5 & 0,3 & $<0,01$ & 4,9 & 2,4 & 9,7 \\
\hline Intercorrência materna & 0,6 & 0,3 & 0,05 & 2,0 & 0,9 & 4,0 \\
\hline
\end{tabular}

\section{Discussão}

Kleinbaum ${ }^{9}$ caracteriza o problema multivariado quando temos um conjunto de variáveis independentes para predizer uma variável dependente. Neste caso, a modelagem matemática é usada para o estudo das complexas relações entre muitas variáveis e a regressão logística pode ser usada para descrevê-las.

Inicialmente, consideramos o papel isolado de cada uma das variáveis estudadas e o que nos informa a literatura. Os limites da idade reprodutiva têm sido considerados de risco para o resultado gestacional. A gestação precoce (adolescência) era classicamente admitida como de risco pela falta de amadurecimento do organismo, que não estaria totalmente apto para o processo. Já a gestação tardia preocupava pelas intercorrências clínicas ou maus antecedentes obstétricos.

A gestação em idade materna avançada ou tardia foi referida como fator de risco para cesariana, sendo que o ponto de corte variou entre 30 e $35 \operatorname{anos}^{10,11}$. Já a adolescência foi valorizada como fator de proteção contra a cesariana ${ }^{12}$. Observou-se que a adolescência (idade menor que 20 anos) comportou-se como fator de proteção e a idade avançada como fator de risco para cesariana. A idade materna aos 35 anos não foi estatisticamente significativa mas o foi aos 28 anos como fator de risco para cesariana. A interpretação é que na medida em que a idade avança, independente dos demais fatores, que estão controlados pelo modelo multivariado, aumenta o risco de cesariana, o que se observou quando a idade materna participou como variável contínua. Do ponto de vista clínico interessa estabelecer um ponto de corte que, classicamente, foi aos 28 anos. Com o avanço da Obstetrícia e melhores condições de saúde das pacientes, este ponto de corte foi deslocado para 35 anos, havendo no entanto polêmica quanto a este ponto. 
A idade gestacional foi relatada como fator relacionado ao aumento da incidência de cesariana quando além da $35^{\mathrm{a}}$ semana ${ }^{13}$. A idade gestacional foi observada como variável contínua para tentar obter-se o maior poder de cálculo. Ainda assim, na análise bivariada, a comparação de médias de idade gestacional entre o grupo de cesariana e o controle não permitiu identificar diferença entre eles.

A paridade é confundida por diversos outros, tais como a idade materna e o antecedente de cesariana, para citar somente dois. Assim, foi observado que a paridade foi confundida pela idade, sendo que a primeira não ficou caracterizada como fator de risco mas, sim, a idade materna avança$\mathrm{da}^{10}$. A paridade, quando controlada para fatores de confusão (idade materna, intercorrências clínicas), tem sido observada como fator de risco para cesariana quando associada ao diabete ou ao longo do tempo ${ }^{13}$. Por outro lado, o antecedente de parto normal foi reconhecido como fator de proteção para a cesariana ${ }^{12}$. No extremo oposto, a multiparidade extrema (10 ou mais partos) foi observada como fator de risco para cesariana ${ }^{14}$, fato não confirmado por outros autores ${ }^{15}$.

$\mathrm{O}$ antecedente de parto normal foi considerado separadamente do antecedente de cesariana. O número de partos normais atuou como fator de proteção. Assim, o risco maior foi para a primipara e foi decrescendo a cada parto normal. A amplitude do número de partos normais foi de 0 a 8, não permitindo inferências quanto à multiparidade extrema.

$\mathrm{O}$ antecedente de cesariana ficou historicamente marcado como fator de risco para cesariana quando Craigin, em 1916, exarou o seu aforisma: "uma vez cesariana, sempre cesariana" ${ }^{1}$. A sentença teve profunda influência na obstetrícia norte-americana e repercute até os dias de hoje. Cabe lembrar que o contexto de 1916 era de cesariana clássica (corporal longitudinal), mais sujeita à rotura uterina. Modernamente, após o advento da incisão uterina segmentar transversa, diversos estudos mostraram que o risco de rotura não é estatisticamente diferente do risco em não portadoras de cicatriz de cesariana, embora haja polêmica.

Limitando a discussão à análise do antecedente de cesariana como fator de risco para uma nova cesariana, aquele foi reconhecido como tal ${ }^{16-}$ ${ }^{18}$, havendo no entanto quem discorde ${ }^{13}$. No presente estudo o antecedente de cesariana foi observado como fator de risco para cesariana, na razão direta do número de cesarianas prévias.

A apresentação fetal eutócica é a cefálica fletida. A apresentação anômala (qualquer outra que não a cefálica fletida) foi reconhecida como fator de risco para cesariana ${ }^{12,19}$. No presente estudo não foram observadas outras apresentações que não a cefálica fletida, utilizada como categoria de referência, e a apresentação pélvica, que revelou-se fator de risco para cesariana. A maior incidência de cesariana na apresentação pélvica tem sido justificada pelo maior risco perinatal. Este risco só ficou devidamente caracterizado pelo recente ensaio clínico de Hannah et al. ${ }^{20}$ em que notou-se maior morbidade e mortalidade perinatal quando a apresentação pélvica foi conduzida pela via vaginal. A prenhez gemelar foi reconhecida como fator de risco para cesariana ${ }^{12}$, fato confirmado pelo presente estudo.

A expressão hemorragia da segunda metade da prenhez englobou a inserção baixa da placenta e o descolamento prematuro da placenta. A placenta prévia (modalidade de inserção baixa da placenta) é fator de risco para cesariana ${ }^{21}$. A placenta prévia foi, ainda, fator de risco para o descolamento prematuro da placenta contribuindo para a confusão entre os dois fatores ${ }^{21}$. No presente estudo a hemorragia da segunda metade da prenhez foi identificada como fator de risco para cesariana.

A amniorrexe prematura pode acompanharse de colo não maduro, quando seria fator de risco para cesariana, o que entretanto não ocorreu no estudo de Hannah et al. ${ }^{22}$. O presente estudo concorda com a literatura citada, não tendo observado a amniorrexe prematura como fator de risco para cesariana.

A hipertensão arterial associada à gravidez sob diferentes formas (doença hipertensiva crônica ou hipertensão específica da gravidez) e graus (pré-eclampsia leve, pré-eclampsia grave e eclampsia) tem sido observada como fator de risco para cesariana ${ }^{13}$. No presente estudo as diversas formas de hipertensão estiveram associadas à maior probabilidade de cesariana, sendo a ordem de risco crescente da pré-eclampsia leve, passando pela hipertensão crônica e culminando com a pré-eclampsia grave, o que é coerente com o modelo teórico de agravamento do risco acompanhando a história natural da doença, vista como um todo. A eclampsia não pôde ser estudada por limitação do estudo, porque apenas cinco pacientes foram portadoras de eclampsia e todas foram submetidas à cesariana. Por falta de grupo de comparação o modelo de regressão rejeitou estas observações. Outras intercorrências clínicas, como o diabete, foram valorizadas como fatores de risco para cesariana ${ }^{19}$, e também reconhecidas nesta pesquisa.

Vista a associação individual de cada fator de risco para cesariana, consideremos agora a análise conjunta (multivariada) que visa ao con- 
trole simultâneo de todos os fatores. Hin et al. ${ }^{8}$, usando regressão logística, construíram dois modelos preditivos de cesariana. A construção do modelo de regressão logística tem como base um modelo biológico (plausibilidade) em que se admite, no estado atual do conhecimento, a associação de um determinado fator com a ocorrência do desfecho (cesariana) e são selecionadas as variáveis independentes (fatores de risco). A próxima etapa consiste na avaliação do efeito do fator sobre a ocorrência do desfecho (critério estatístico). Finalmente, associando-se os dois critérios (biológico e estatístico), utilizamos técnica de análise multivariada, no caso a regressão logística, por ser o desfecho do tipo categórico dicotômico (cesariana, sim ou não), conforme orientação de Kleinbaum ${ }^{9}$. Hin et al. ${ }^{8}$ utilizaram um primeiro modelo com variáveis anteparto e outro com acréscimo de variáveis intraparto. Ambos os modelos apresentaram concordância alta entre o predito (probalidade de cesariana) e o observado (ocorrência de cesariana), com $82 \%$ de área sob curva ROC para o modelo intraparto e $81 \%$ para o modelo anteparto. A diferença entre os modelos é que o segundo inclui variáveis só identificáveis no decurso do trabalho de parto.

O presente estudo, utilizando somente variáveis identificadas antes do trabalho de parto, identifica-se com o modelo anteparto de Hin et al. ${ }^{8}$ e permitiu a construção de modelo preditivo de cesariana, tendo alcançado concordância entre $\mathrm{o}$ predito e o observado de $82,3 \%$, podendo ser aceito como estudo de alta acurácia.

O modelo é útil no cálculo da probabilidade de cesariana em cada paciente, considerando-se o seu perfil (idade materna, intercorrência clínica, intercorrência obstétrica). A partir do cálculo da probabilidade de cesariana para determinada paciente podemos selecionar aquelas que apresentavam baixa probabilidade de cesariana e ainda assim foram operadas.

O modelo apresenta pelo menos três aplicações práticas: 1) avaliação da qualidade da assistência obstétrica pela análise das pacientes que tinham probabilidade baixa de cesariana e foram operadas. Por que foram operadas?; 2) no planejamento da assistência obstétrica, por servir de instrumento de orientação da paciente no sistema de saúde, encaminhando-a para nível de assistência compativel com o seu risco de cesariana; 3) para avaliação do impacto de intervenções. Por exemplo, consideremos a portadora de apresentação pélvica. Seu risco de cesariana exclusivamente pela apresentação pélvica é de $31,34 \%$ (resultado da solução da equação representativa do modelo logístico). Ao propormos o pro- grama de transformação da apresentação pélvica em cefálica, qual seria a probabilidade de cesariana, na ausência da apresentação pélvica? Mais uma vez, a solução da equação informa que seria de $9,86 \%$.

Concluindo, foram, então, identificados 12 fatores associados ao risco de cesariana, dos quais dois de proteção (idade menor que 20 anos e antecedente de parto normal) e dez de risco, sendo que nove estiveram de acordo com a maior parte da literatura, a saber: idade materna avançada, antecedente de cesariana, apresentação pélvica, prenhez gemelar, hemorragia da segunda metade da prenhez, pré-eclampsia leve, préeclampsia grave, hipertensão arterial crônica e outras intercorrências maternas. A assistência pré-natal, de efeito variável pela literatura, foi pelo estudo identificada como fator de risco, melhor interpretada como fator de confusão pelo critério biológico.

\section{ABSTRACT}

Purpose: to investigate antepartum factors related to cesarean section and develop a cesarean section predictive model.

Methods: the study design was a retrospective cohort which included all the cared 843 deliveries in a third level unit from June 1993 through November 1994. Children with 1,000 $\mathrm{g}$ birthweight and above were included. The dependent variable was cesarean section (c-section). Independent variables were antepartum factors related to c-section. Logistic regression was used to develop a predictive model. Results: our model showed risk of c-section according to the following variables: maternal age under 20 years $(O R=$ $0.396)$ and over 28 years $(O R=2.133)$; previous vaginal deliveries $(O R=0.626)$; previous c-section $(O R=4.576)$; prenatal care $(O R=2.346)$; breech presentation $(O R=$ $4.174)$; twin pregnancies $(O R=14.065)$; late obstetrical hemorrhage $(O R=28.189)$; mild preeclampsia $(O R=2.180)$; severe preeclampsia $O R=16.738$; chronic hypertension $O R=4.927$ and other clinical problems $(O R=2.012)$. The predictive model had a concordance of $82.3 \%$ between probabilities and responses.

Conclusions: our study identified 12 antepartum factors related to c-section. It was possible to develop a cesarean section predictive model taking into account all previously identified antepartum risk factors.

KEY WORDS: Cesarean section. Predictive model. Pregnancy complications. Delivery, mode of. 


\section{Referências}

1. Cunningham FG, McDonald PC, Leveno KJ, et al. Williams obstetrics. 20th ed. Stamford: Appleton \& Lange; 1997. 1448p.

2. Depp R. Cesarean delivery. In: Gabbe SG, Niebyl JR, Simpson JL, editors. Obstetrics Normal and Problem Pregnancies. 3rd ed. New York: Churchill Livingstone; 1996. p.561-642.

3. Socol ML, Peaceman AM. Active management of labor. Obstet Gynecol Clin North Am 1999: 26:287-94.

4. Notzon FC, Placek PJ, Taffel SM. Comparisons of national cesarean section rates. $\mathrm{N}$ Engl J Med 1987; 316:386-9.

5. Camano L, Vigorito NM, Mattar R. Aumento da freqüência da cesariana. Análise da percentagem verificada na Escola Paulista de Medicina. J Bras Ginecol 1984; 94:407-10.

6. FEBRASGO. Obstetras definem ações do Projeto Maternidade Segura. J FEBRASGO 1994; 1:6-7.

7. Cunha AA, Portela MC, Amed AM, Camano L. Incidência de cesariana: um problema? Femina 2000; 28:553-6.

8. Hin LY, Lau TK, Rogers M, Chang AM. Antepartum and intrapartum prediction of cesarean need: risk scoring on singleton pregnancies. Obstet Gynecol 1997; 90: 183-6.

9. Kleinbaum DG. Logistic regression: a self-learning text. $1^{\text {st }}$ ed. New York: Springer-Verlag; 1994. $282 \mathrm{p}$.

10.Gordon D, Milberg J, Daling J, Hickok D. Advanced maternal age as a risk factor for cesarean delivery. Obstet Gynecol 1991; 77:493-7.

11.Peipert JF, Bracken MB. Maternal age: an independent risk factor for cesarean delivery. Obstet Gynecol 1993; 81:200-5.

12.Amini SB, Catalano PM, Dierker LJ, Mann LI. Births to teenagers: trends and obstetric outcomes. Obstet Gynecol 1996; 87:668-74.
13.Elferink-Stinkens PM, Brand R, Van-Hemel OJ. Trends in caesarean section rates among highand medium-risk pregnancies in The Netherlands 1983-1992. Eur J Obstet Gynecol Reprod Biol 1995; 59:159-67.

14.Fayed HM, Abid SF, Stevens B. Risk factors in extreme grand multiparity. Int J Gynaecol Obstet 1993; 41:17-22.

15.Kaplan B, Harel L, Neri A, Rabinerson D, Goldman GA, Chayen B. Great grand multiparity: beyond the 10th delivery. Int $\mathrm{J}$ Gynaecol Obstet 1995; 50:17-9.

16.Beckley S, Gee H, Newton JR. Scar rupture in labour after previous lower uterine segment caesarean section: the role of uterine activity measurement. Br J Obstet Gynaecol 1991; 98:265-9.

17.Norman P, Kostovcik S, Lanning A. Elective repeat cesarean sections: how many could be vaginal births? CMAJ 1993; 149:431-5.

18.Adair CD, Sanchez-Ramos L, Gaudier FL, Kaunitz AM, McDyer DC, Briones D. Labor induction in patients with previous cesarean section. Am J Perinatol 1995; 12:450-4.

19.Bernstein IM, Catalano PM. Examination of factors contributing to the risk of cesarean delivery in women with gestational diabetes. Obstet Gynecol 1994; 83:462-5.

20.Hannah ME, Hannah WJ, Hewson SA, Hodnett ED, Saigal S, Willan AR. Planned caesarean section versus planned vaginal birth for breech presentation at term: a randomised multicentre trial. The Term Breech Trial Collaborative Group. Lancet 2000; 356:1375-83.

21.Iyasu S, Saftlas AK, Rowley L, Koonin LM, Lawson HW, Atrash HK. The epidemiology of placenta previa in the United States, 1979 through 1987. Am J Obstet Gynecol 1993; 168:1424-9.

22.Hannah ME, Ohlsson A, Farine D, et al. Induction of labor compared with expectant management for prelabor rupture of the membranes at term. N Engl J Med 1996; 334:1005-10.

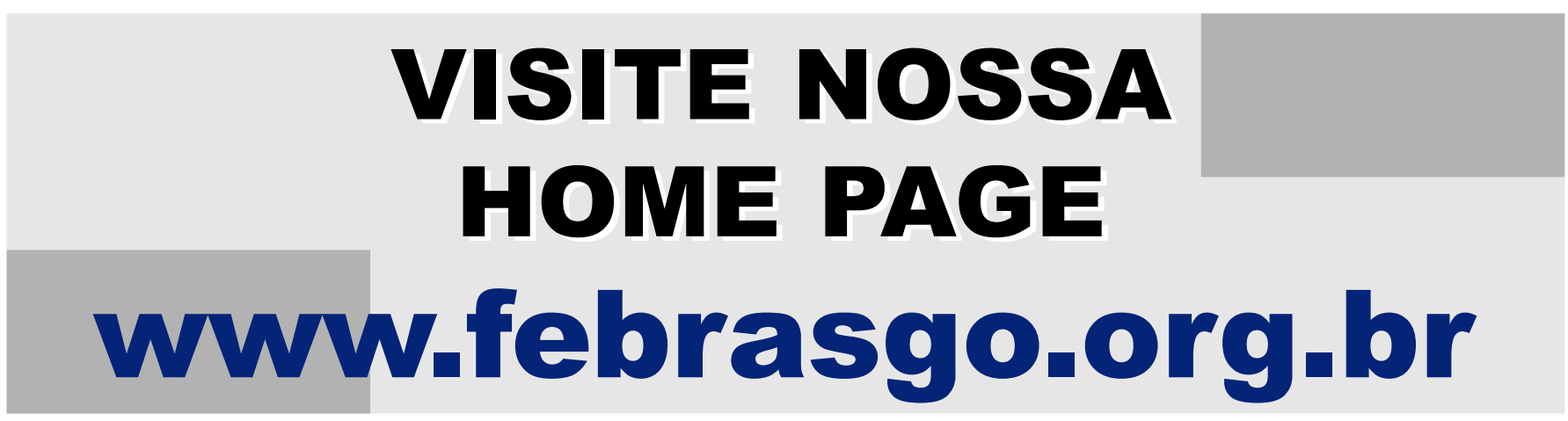

